\title{
Achieving competitive, customized employment through specialized services (ACCESS)
}

\author{
Tammy Jorgensen Smith*, Deveney Ching, Amanda Weston and Christina J. Dillahunt-Aspillaga \\ Department of Rehabilitation and Mental Health Counseling, College of Behavioral and Community \\ Sciences, University of South Florida, Tampa, FL, USA
}

Revised/Accepted November 2018

\begin{abstract}
.
BACKGROUND: There is a paucity of research regarding employment services for adults with autism. Interventions typically focus on adapting behaviors of the person rather than customized approaches and environmental adaptations that may improve employment matching and ongoing, competitive employment rates.

OBJECTIVE: The ACCESS intervention protocolizes the customized employment (CE) process and has the potential to optimize employment outcomes in adults with autism. Lessons learned during the implementation of the Phase I open trial are presented.

METHODS: The study utilizes a randomized pre-test post-test experimental control group design. Phase I - open trial $(N=10)$ of the study tested feasibility and acceptability of the ACCESS intervention. Phase II - randomized controlled trial $(N=30)$ will test preliminary efficacy of an amended intervention protocol.

RESULTS: Findings from the open trial suggest the need for a revised recruitment strategy and an extended timeframe for the intervention. Thorough screening of practitioners and assessing participants' work motivation may reduce attrition.

CONCLUSION: An evidence-based, user-friendly process that employs well-trained practitioners is critical to successful implementation of CE services with fidelity and consistency. A collaborative approach to training, implementation, documentation, evaluation, and pre and post-employment supports is essential to promote an effective and sustainable intervention.
\end{abstract}

Keywords: Autism, customized employment, social capital, self-determination

\section{Introduction}

Autism spectrum disorders (ASD) are a group of neurodevelopmental conditions whose defining features include challenges in social communication and

\footnotetext{
*Address for correspondence: Tammy Jorgensen Smith, Ph.D., CRC, Associate Professor, Department of Rehabilitation and Mental Health Counseling, University of South Florida, College of Behavioral and Community Sciences, 13301 Bruce B. Downs Blvd, MHC 1632-12, Tampa, FL 33612, USA. Tel.: +1 813974 0973; Fax: +1 813974 8080; E-mail: tjsmith@bcs.usf.edu.
}

interaction across contexts and restrictive, repetitive patterns of behavior, interest, or activities (American Psychological Association [APA], 2013). Experts recognize a vast heterogeneity of both abilities and challenges in individuals with autism (Shattuck et al., 2012). Manifestations of core diagnostic features vary widely across and within individuals over time (Happe \& Charlton, 2012).

The prevalence of ASD is 1 in 59 (Centers for Disease Control and Prevention [CDC], 2018), a significant increase from 2000 (1 in 150) which 
reflects increases in diagnostic criteria, awareness, and evolving reporting standards (Christensen et al., 2016; Newschaffer et al., 2007; Williams, Higgins, $\&$ Brayne, 2006). One prevalence characteristic that has remained relatively constant is the estimated ratio of approximately 4.5 male: 1 female with ASD (Christensen et al., 2016). Autism is second only to intellectual disabilities (ID) as the most common severe developmental disability (DD) in the US (Newschaffer et al., 2012).

Autism is a life-long condition with a life expectancy only three years shorter than the general population (Brugha et al., 2011; Levy \& Perry, 2011; Lin, Lin, \& Wu, 2009; Marriage, Wolverton, \& Marriage, 2009). Functional skills of persons on the autism spectrum often plateau during adulthood (Shattuck et al., 2011; Smith, Maenner, \& Seltzer, 2012; Taylor \& Seltzer, 2011). As few as 25\% access postsecondary education, maintain competitive employment, live independently, and/or sustain social relationships (Fein et al., 2009; Helt et al., 2008; Howlin \& Moss, 2012; Levy \& Perry, 2011; Seltzer, Shattuck, Abbeduto, \& Greenberg, 2004).

\subsection{Employment challenges and risk factors for adults with autism}

Research shows only $4-11 \%$ of persons on with ASD are competitively employed (Cerdurland et al., 2008; Taylor \& Seltzer, 2011; Wehman et al., 2014; Wilczynski, Tramell, \& Clarke, 2013). Many employed persons on the autism spectrum are working part-time, underemployed, underpaid, or in sheltered workshops (Beadle-Brown, Murphy, \& Wing, 2005; Keogh, Bernheimer, \& Guthrie, 2004; O'Brien, 2006; Roux et al., 2013). Competitive employment, defined as employment in an inclusive, community-based setting at a wage comparable to others in the same position, provides substantial economic benefits to society because employed individuals pay taxes and rely less on government-funded programs. Additionally, "employment plays a critical role in social, emotional, and financial well-being for people with ASD" (Nord et al., 2016, p.11).

Major risk factors for unemployment in persons on the autism spectrum include co-occurring conditions (Ghaziuddin \& Zafar, 2008; Holweda et al., 2007; Perkins \& Berkman, 2012; Simonoff et al., 2008; Skokauskas \& Gallagher, 2010; Mannion, Leader, \& Healy, 2013), oppositional behaviors and lower speech/language ability (Wehman et al., 2014), and social communication challenges (Hagner \& Cooney,
2005; Hillier et al., 2007; Patterson \& Rafferty, 2010). These may include issues understanding directions or inferring meaning from facial expressions or voice tone and frustration that individuals with autism express when their attempts to communicate are unsuccessful (Hurlbutt \& Chalmers, 2004; Matson et al., 2011; McClean \& Grey, 2007).

\subsection{Current clinical practice}

Most VR agencies utilize standardized assessments to evaluate vocational potential of job seekers, regardless of the type of disability (Hagner, 2010; Jepsen, 1994). Experts note this approach: (1) fails to identify strengths the individual brings to the workplace (Sodak, 2000), (2) focuses on single job placement rather than ongoing job success, and (3) engenders feelings of disempowerment in the person (Cooney, 2002; Grasso, Jitendra, Browder, \& Harp, 2004; Sodak, 2000; Ysseldyke \& Olsen, 1999). Traditional vocational evaluations are prescribed and do not typically accommodate for the unique needs and challenges of people on the autism spectrum, nor are their results normed for persons with disabilities which prevents them from providing accurate, comprehensive information. VR experts are increasingly recognizing the value of strengths-based assessments that focus on performance of real-world tasks in natural contexts to identify knowledge, skills, and aptitudes that traditional assessments do not evaluate (Gulikers, Bastiaens, \& Kirschner, 2004; Maclellan, 2004).

\subsection{Critical components in effective employment interventions for adults with autism}

\subsubsection{Employment matching}

Skills required in different work settings vary considerably, as do the skills that adults with autism bring to the job market. Thus, it is critical to match job requirements to interests and talents of the job seeker. Rehabilitation professionals must explore the preferences, skills, and support needs of adults with autism through observing them in real life situations, using their connections to find the best job matches (social capital), and promoting natural supports in the workplace (Callahan, Shumpert, \& Condon, 2009; Griffin, Hammis, \& Geary, 2007; Luecking, Fabian, \& Tilson, 2004; Migliore, Butterworth, \& Zalewska, 2014). A leading approach to employment matching is the Discovery process. By meeting with the individual, family, friends, and support staff and observing the 
individual's skills and interests in a natural environment, a VR practitioner 'discovers' both the strengths and challenges faced by the job seeker, replete with complexities seldom captured through psychometric testing, interest interviews, or structured evaluations (Griffin, Hammis, Geary, \& Sullivan, 2008). Utilizing this information, the VR practitioner produces a robust narrative profile to guide employment matching (Callahan, Griffin, \& Hammis, 2011).

\subsubsection{Employment customization}

$\mathrm{CE}$ includes negotiating employment opportunities with employers through identification of unmet business needs that may be fulfilled by the talents of a job seeker. This process does not begin with a response to an advertisement for employees, but rather through meeting with employers and touring businesses to identify mutually beneficial matches. Challenges that job seekers face can be managed by adapting the workplace to accommodate the person based on his or her unique support needs. A target for environmental adaptation is knowledge and attitudes of employers/coworkers regarding autism. This can be addressed through onsite and sustainable web-based training options that are easily updated and on-demand to meet the ongoing training needs of businesses. Potential solutions to employment concerns for adults on the autism spectrum include simplified job descriptions, clear expectations, reminders, feedback, reassurance, and consistent schedules (Hendricks, 2010; Migliore, Butterworth, \& Zalewska, 2014). Organizing work activities/environments in ways that assist the person in initiating activities, staying on task, and knowing when to transition to new tasks can be helpful when the adaptations are compatible with how the person processes information (Migliore, Butterworth, \& Zalwska, 2014; Taylor \& Seltzer, 2011).

\section{Customized Employment and the ACCESS Model}

The CE process is grounded in social-ecological theory in that it acknowledges the complex interplay between a person and the environment. It emphasizes the importance of person-environment fit as well as the diverse systems that influence human functioning (Schalock et al., 2010; Shogren, 2013). Unique aspects of each person such as age, type of disability, functional capacities, disposition, and interaction style and contextual factors (e.g. living arrangements, geographical location, support systems, connections, resources) that align with individual interest and talents are considered along with conditions that may impact successful, long-term employment. Social capital also plays an important role in the CE process as networks and connections assist in understanding preferences, skills, and support needs of the job seeker and provide insight into potential employment opportunities within the community.

$\mathrm{CE}$ strategies are being increasingly adopted by VR programs across the country due to recent legislation that specifically identifies $\mathrm{CE}$ as an employment outcome under the public VR program (Section $\S 361.5(c)(15)$ - USDOL, 2014b; H.R. 803, 2017) and because of 'real-world' evidence that CE strategies improve competitive, integrated employment outcomes in people with complex disabilities. The CE process, which has the job seeker at its core, and consists of the following key elements: 1) Discovery process, 2) vocational profile, 3) CE planning meeting, 4) portfolio/visual resume, 5) customized job development and negotiation, and 6) accommodations and post-employment supports. Table 1 provides a brief description of the elements of the $\mathrm{CE}$ process.

Table 1

CE Process Elements and Descriptions

\begin{tabular}{|c|c|}
\hline PROCESS ELEMENT & DESCRIPTION \\
\hline Discovery Process & Qualitative assessment of interests, skills, and conditions for employment. \\
\hline Vocational Profile & $\begin{array}{l}\text { Robust, strengths-based narrative snapshot of the job seeker and translation of "discovered" interests and } \\
\text { skills to potential employment opportunities. }\end{array}$ \\
\hline CE Planning & $\begin{array}{l}\text { Person-centered team approach (job seeker, family, friends, VR counselor, other service/and support } \\
\text { providers) to develop a blueprint to employment. }\end{array}$ \\
\hline Visual Resume & $\begin{array}{l}\text { Video/photo resume illustrating job seeker's ability to complete essential elements of a job. Included in a } \\
\text { career portfolio. }\end{array}$ \\
\hline $\begin{array}{l}\text { Job Development and } \\
\text { Negotiation }\end{array}$ & $\begin{array}{l}\text { Working with employers to identify unmet needs and to customize job opportunities to benefit from job } \\
\text { seeker skills and talents. }\end{array}$ \\
\hline $\begin{array}{l}\text { Accommodations and } \\
\text { Employment Supports }\end{array}$ & $\begin{array}{l}\text { Environmental restructuring, training, and customization to enhance job seeker success and employment } \\
\text { retention. }\end{array}$ \\
\hline
\end{tabular}


ACCESS protocolizes the CE process within a user-friendly system that incorporates an interconnected package of tools, templates, and logs that promote consistent application of the intervention. Elements from a myriad of popular CE models and feedback from stakeholder groups were referenced in the development of the ACCESS intervention. The model is further informed by The Essential Elements of Customized Employment for Universal Application (2017), a document developed by leaders in the field and published by the Workforce Innovation Technical Assistance Center (WINTAC). Additionally, a fidelity instrument, the Benchmarks of Quality Checklist (BQC), has been developed to 1) facilitate training and guidance for community-based providers who implement the ACCESS intervention; 2) promote adherence to the ACCESS model; and 3) document omissions, additions, and adaptations that are made during the implementation process.

The purpose of this article is to present preliminary findings and lessons learned from the Phase I, open trial $(N=10)$ of the ACCESS study. This information will be utilized to inform the Phase II - randomized controlled trial $(\mathrm{RCT})(N=30)$ that will test preliminary efficacy of an amended intervention protocol. Pilot data will inform the development of an application for a larger RCT through a NIH/NIMH-R01 mechanism.

\section{Methods}

\subsection{Research design}

The ACCESS study utilizes a randomized pretest post-test experimental control group design. The research team (RT) conducted an open trial (Phase I) to test the feasibility and acceptability of the ACCESS intervention. The open trial $(N=10)$ is being evaluated by pre-post participant data and open-ended practitioner and participant interviews. In Phase II, the RT will test the revised ACCESS protocol in a RCT $(N=30)$ with adults on the autism spectrum being randomly assigned to the usual care or ACCESS intervention. Usual care conditions allow the researchers to examine outcomes of adults with autism who receive no services due to ineligibility determinations, waitlists, or other factors. The RCT will be evaluated by pre-post assessments and 3 and 6-month follow-up data.

\subsection{Specific aims}

Primary aims of the ACCESS study are to: 1) refine training, treatment and study protocols to test the ACCESS intervention; 2) examine the feasibility and acceptability of ACCESS in an open trial; and 3) examine the feasibility, acceptability and preliminary effects of ACCESS relative to usual care in a RCT of 30 adults with autism. After iteratively improving training, treatment, and study protocols, the (RT) expects to gather convincing evidence of the feasibility and effectiveness of the ACCESS intervention to support a larger RCT delivered by certified VR practitioners to adults on the autism spectrum.

\subsection{Participant recruitment}

Inclusionary criteria include diagnosis of autism, age $22+$, moderate speech capacity determined by discourse competence defined as ability to connect sentences to form a meaningful whole out of a series of utterances, moderate intellectual capacity determined by a score of 70 or greater on the Kaufman Brief Intelligence Test (KBIT-2)(2004), and English as the primary language. Exclusionary criteria include history of harm to self or others.

The RT recruited from a regional autism center's database that contains approximately 1,228 adults with ASD within its 14-county service area. A pilot survey was emailed to 418 eligible center constituents to test the feasibility of recruitment. In one wave of distribution, $92 \%$ of respondents $(n=48)$ indicated interest in participating in the study.

At the start of Phase I of the ACCESS study, the autism center emailed an announcement to eligible constituents with contact information for learning more about the project. In follow up, the RT provided additional information and addressed questions and concerns presented by potential participants. Eligible individuals were scheduled to meet with the RT to review and sign the institutional review board (IRB) approved informed consent form and complete baseline assessments.

\subsection{Practitioner recruitment}

Community-based rehabilitation practitioners who currently provide CE services to eligible participants of the public VR system were recruited to the study. To become certified to deliver CE strategies, practitioners must complete a 14-week, intensive 
training that includes eight performance-based content modules and an experiential component with a VR job seeker to translate content knowledge into practice with expert technical assistance. Practitioners are required to score $80 \%$ or higher on each course module and receive approval of vocational profile and CE blueprint prior to becoming certified to provide the $\mathrm{CE}$ services.

\subsection{Instrumentation}

Table 2 provides a list of participant measures with their associated constructs and time points for administration. These assessments are administered by the RT pre and post intervention; however, results are not shared with practitioners to prevent the quantitative scores/data from influencing the qualitative process of Discovery. Practitioner measures are included in Table 3.

\subsubsection{Risk factors}

- KBIT-2 assesses cognitive functioning (Kaufman \& Kaufman, 2004). It generates verbal, non-verbal, and composite IQ standard scores $(M=100, S D=15)$ and has very strong convergent validity with Wechsler Adult Intelligence Scale 3rd ed. test scores.

- Autism Spectrum Quotient is a 50-item self and parent report scale that measures autism traits along five sub-scales: social skills, attention shifting, attention to detail, communication, and imagination (Baron-Cohen, Wheelwright, Skinner, Martin, \& Clubley, 2001). Internal consistency is estimated to be 0.82 with test-retest reliability of 0.70 in adults with ASD.

\subsubsection{Functional outcomes}

- Social Responsiveness Scale TM, 2nd Edition, (SRS-2) Adult Self-Report Form is a 65-item rat-

Table 2

Participant Measures

\begin{tabular}{|c|c|c|c|c|}
\hline MEASURE & CONSTRUCT & ITEMS & PSYCHO-METRICS & TIME POINTS \\
\hline Demographics & $\begin{array}{l}\text { Age, gender, race/ethnicity, work } \\
\text { history, education level, SES, } \\
\text { living situation }\end{array}$ & - & N/A & B \\
\hline \multirow[t]{2}{*}{ Risk Factors } & Cognitive functioning & 97 & KBIT-2 & B \\
\hline & Level of ASD & 50 & AQ & B \\
\hline \multirow{2}{*}{$\begin{array}{l}\text { Functional Outcomes } \\
\text { (social, communication, } \\
\text { attention, mannerisms, } \\
\text { self-determination) }\end{array}$} & Level of Social Challenges & 65 & SRS-2 & $\mathrm{B}, \mathrm{P}$ \\
\hline & Self-determination & 51 & GCOS & $\mathrm{B}, \mathrm{P}$ \\
\hline Employment Outcomes & Competitive Employment & - & N/A & $\mathrm{B}, \mathrm{P}, 3,6$ \\
\hline Secondary Outcomes & Quality of Life & 40 & QOL-Q & $\mathrm{B}, \mathrm{P}$ \\
\hline
\end{tabular}

SES = Socioeconomic status; KBIT-2 = Kaufman Brief Intelligence Test; AQ = Autism Quotient; SRS-2= Social Responsiveness Scale; GCOS = General Causality Orientations Scale; QOL-Q=Quality of Life Questionnaire; B = Baseline; $P=$ Post-intervention; 3 and $6=3$ and 6 months post-intervention.

Table 3

Practitioner Measures

\begin{tabular}{lllc}
\hline MEASURE & CONSTRUCT & RATER & TIME POINTS \\
\hline $\begin{array}{l}\text { DESCRIPTORS } \\
\begin{array}{l}\text { Age, gender, } \\
\text { race/ethnicity, } \\
\text { education level }\end{array}\end{array}$ & Demographics & Practitioner & B \\
& VR training & Practitioner & \\
& VR experience & Practitioner & B \\
& ASD experience & Practitioner & B \\
& Languages & Practitioner & B \\
TREATMENT DATA & Fidelity & Qualitative Scientist & P \\
& Training time & PI, Co-PI, RAs & P \\
& Tx time/participant & PI, Co-PI, RAs & P \\
\hline
\end{tabular}

$\mathrm{B}=$ Baseline; $\quad P=$ Post-Intervention; $\quad \mathrm{VR}=$ Vocational Rehabilitation; $\mathrm{ASD}=$ Autism Spectrum Disability; Tx = treatment; $\mathrm{PI}=$ Principal Investigator; Co-PI =Co-Principal Investigator; RA $=$ Research Assistant. 
ing scale of the level of ASD symptoms in natural social settings measuring social awareness, social information processing, capacity for reciprocal social communication, social avoidance, and autism mannerisms (Constantino, 2005). Internal consistency is estimated to be.93-.97, with evidence of discriminant, concurrent, convergent and construct validity (Granader et al., 2010).

- General Causality Orientations Scale (GCOS) is a 51-item self-report measure of self-determination designed for use with persons with disabilities (Deci \& Ryan, 1985). The scale assesses three motivational orientations within an individual - autonomy (intrinsic motivation), controlled (motivated by rewards, deadlines, etc.), and impersonal (external locus of control; luck or fate). This 17-vignette version of the instrument also measures social-interactions rather than solely measuring achievements. The scale has been shown to be reliable, with Cronbach alphas of.75 and a test-retest coefficient of. 74 .

\subsubsection{Employment outcomes}

- Competitive Employment (primary outcome) Participants complete a structured interview with the RT at 3 and 6-months post-intervention. Data captured includes post-intervention employment, employment type, current employment status, job title(s), wage(s), average hours per week, supports, transportation method, and challenges.

\subsubsection{Secondary outcomes}

- Quality of Life Questionnaire (QOL-Q) is a 40item measure of objective and subjective quality of life (QOL) in persons with DD (Schalock \& Keith, 1993). Four factors including satisfaction, competence/productivity, empowerment/ independence, and social belonging/community integration are measured. Internal consistency is estimated at.90. Inter-rater reliabilities are reported between.73-.83 with a test-retest coefficient of.87.

\section{Analyses}

\subsection{Phase I}

Feasibility is evaluated by determining whether the RT can meet the recruitment, intervention, and data collection benchmarks. Recruitment feasibility is demonstrated by 16 interested participants identified in a two-month period, 13 of whom meet eligibility criteria and 10 of whom will agree to be participants in the study (permitting a dropout rate of approximately $25 \%$ ). Baseline data collection feasibility is demonstrated by $80 \%$ of participants completing all baseline instruments with no more than $10 \%$ missing data on any one instrument. Intervention feasibility is demonstrated by practitioners delivering the ACCESS intervention to $90 \%$ of participants with fidelity to the treatment protocol. Follow-up data collection feasibility will be demonstrated by $80 \%$ of treatment completers completing all follow-up instruments with no more than $10 \%$ missing data on any instrument. Acceptability will be demonstrated by at least $60 \%$ of participants completing all treatment and supplemental assessments.

Interview data will be analyzed using a pattern matching technique that compares observed data with a theoretically predicted pattern (Yin, 2003). Systematic coding of text is a key element in qualitative data analysis as codes represent the underlying assumptions of the analysis (Yin, 2003). An initial set of codes representing the research domains will be identified and defined a priori. A codebook will be developed to include code names, definitions, inclusion criteria, exclusion criteria, and an example of passage that illustrates how the code may be presented within the text codebook (Miles \& Huberman, 1994). New codes will be developed should a previously unidentified theme arise during analyses of data. A strategy of seeking alternative explanations for patterns observed in the data will be used to strengthen internal validity and build the explanatory power of the qualitative data (Guest \& MacQueen, 2008).

Phase II data analyses will be presented in a future publication once the study is completed and associated data is available.

\section{Results}

\subsection{Preliminary data-Phase I}

Phase I data is currently limited to descriptive data while post intervention assessments and interviews are in process. The enrollment period was extended from two months to five months due to a hurricane that disrupted progression. A total of 13 individuals (10 males and 3 females) consented to participate in the study and completed the baseline assessments. All but one individual, who did not meet eligibility 
requirements, were enrolled in the study. Initial (baseline) feasibility benchmarks were achieved. However, acceptability benchmarks have not been met with only four participants completing the open trial. This can be attributed, in part, to one practitioner who was not successful with any of her randomly assigned participants $(n=5)$. The remaining participant did not withdraw from the study, but rather placed the process on hold temporarily while a benefits analysis is conducted.

Most participants were between the ages of 23-25 $(n=6)$. The remaining participants $(n=7)$ included one participant each in the 26-28 and 29-31 age ranges, two in the 32-34 age range, and three in the 35-37 age range. None of the participants were over the age of 37 or under the age of 23. Ten of the participants are White with the other three identifying as Black ( $n=1)$, Asian $(n=1)$, and Latino $(n=1)$.

Nine participants reported a paid work history; the other four have volunteered previously. All participants completed high school - six with a regular diploma, two with a special diploma, and one with a general education development (GED). Two participants completed community college and two have earned a bachelor's degree.

Self-reported socioeconomic statuses ranged from less than $\$ 10,000$ annually $(n=7)$ to between $\$ 10,000$ to $\$ 20,000$ annually $(n=5)$. The remaining participant reported "not sure". None of the participants live independently in their own home. Two participants rent a home or apartment with friends or roommates. Most participants $(n=9)$ live with family.

\subsection{Lessons learned}

A pre-study recruitment survey indicated plenty of demand by eligible individuals. When we began actual recruitment, very few responded. The original recruitment strategy was via a flyer sent by email to over 900 eligible individuals through a regional autism center's database. We have revised the recruitment strategy, for Phase 2 (RCT), to conduct information sessions throughout the recruitment area after learning that potential participants do not want to sign up without being able to have questions answered by the research team. The recruitment flyer was also updated based on feedback from Phase I stakeholders.

After several participants withdrew from the study and based on information obtained through qualitative interviews (by unbiased scientists) with these participants, we have added a work motivation assessment that includes the participant's definition of a successful outcome. It seems that the motivation for participating was not intrinsic for a couple of the participants but, reported in post interviews as being influenced by family member.

We also learned that one practitioner, who was randomly assigned half $(n=5)$ of the Phase I participants, was not successful with any of the participants she served. Most participants only met with this practitioner one or two times prior to withdrawing from the study. Other practitioners have not experienced the same challenges leading the RT to hypothesize the attrition may be attributed to the specific practitioner rather than the intervention.

Demonstration projects indicate the typical timeframe for the CE process is $4-6$ months. We have learned that the average time is closer to 8-9 months for individuals with ASD. This is due, in part, to complexities that extend the time needed for a robust Discovery process. Other contributors to the extended timeframe include competing work priorities for practitioners, scheduling issues between the practitioners and participants, and turnover in practitioners.

\section{Conclusions}

\subsection{Implications for policy and practice}

- The Workforce Innovation and Opportunity Act (WIOA)(USDL, 2014) requires VR agencies to integrate $\mathrm{CE}$ strategies into their menu of services. An evidence-based, user-friendly CE process (such as ACCESS) is critical to successful implementation of $\mathrm{CE}$ services in a consistent manner across states.

- Success of the ACCESS study, and other CE projects, is greatly impacted by provider agencies and practitioners. Highly qualified, well-trained practitioners are necessary for successful implementation. High turnover in provider agencies paired with large caseloads/capacity issues are ongoing issues - particularly when serving individuals with complex disabilities.

- Successful integration of CE strategies into the VR system will require involvement by all stakeholders (individuals, families, provider agencies/ practitioners, VR personnel, policymakers, etc.). A collaborative approach to training, process, documentation, and employment supports is essential to effectiveness and sustainability of the intervention. 


\subsection{Goals for future work}

If feasibility, acceptability and preliminary effectiveness of ACCESS is supported, the RT will develop an R01 application to conduct a larger RCT of the ACCESS intervention. Specifically, the RT proposes to power the RCT to evaluate whether ACCESS achieves the recommended effect size over the control intervention reflecting intervention effectiveness. In addition to estimating a definitive effect size, greater resources available through an R01 mechanism will allow the team to test postulated mediators of treatment outcomes using lagged analyses and recruit representative samples from under-represented groups.

\section{Acknowledgments}

Research reported in this publication is supported by the National Institute of Mental Health of the National Institutes of Health under Award Number R34MH111556. The content is solely the responsibility of the authors and does not necessarily represent the official views of the National Institutes of Health. The authors would like to acknowledge the organizations and people who make this research possible. These include the Center for Autism and Related Disabilities at the University of South Florida for assisting with participant recruitment; the collaborating community-based provider agencies (The Learning Academy, The Grow Group and The Diversity Initiative); the study participants; and the rest of the research team - Drs. Wei Wang, Sharon Hodges, Lodi Rohrer, and Flandra Ismajli.

\section{Conflict of interest}

The authors declare that they have no conflict of interest.

\section{Ethics statement}

Procedures involving experiments on human subjects are done in accord with the ethical standards of the Committee on Human Experimentation of the institution in which the experiments were done or in accord with the Helsinki Declaration of 1975.

\section{References}

American Psychiatric Association. (2013). Diagnostic and statistical manual of mental disorders. (5th ed.). Arlington, VA.

Baron-Cohen, S., Wheelwright, S., Skinner, R., Martin, J., \& Clubley, E. (2001). The autism-spectrum quotient (AQ): Evidence for Asperger syndrome/high-functioning autism, males and females, scientists and mathematicians. Journal of Autism and Developmental Disorders, 31, 5-7. Retrieved from http://docs.autismresearchcentre.com/papers/2001_BCetal_ AQ.pdf

Beadle-Brown, J., Murphy, G., \& Wing, L. (2005). Long-term outcome for people with severe intellectual disabilities: Impact of social impairment. American Journal of Mental Retardation, 110, 1-12.

Brugha, T. S., McManus, S., Bankart, J., Scott, F., Purdon, S., Smith, J., ...\& Meltzer, H. (2011). Epidemiology of autism spectrum disorders in adults in the community in England. Archives of General Psychiatry, 68, 459-465. doi: 10.1001/archgenpsychiatry.2011.38

Callahan, M., Shumpert, N., \& Condon, E. (2009). Discovery: Charting the course to customized employment. Gautier, MS: Marc Gold \& Associates.

Callahan, M., Griffin, C., \& Hammis, D. (2011). Twenty years of employment for persons with significant disabilities: A retrospective. Journal of Vocational Rehabilitation, 35, 163-172. doi: 10.3233/JVR-2011-0565

Cedurland, M., Hagberg, B., Billstedt, E., Gillberg, I. C., \& Gilberg, C. (2008). Asperger syndrome and autism: A comparative longitudinal follow-up study more than 5 years after original diagnosis. Journal of Autism and Developmental Disorders, 38, 72-85.

Centers for Disease Control and Prevention (CDC). (2018). Data and Statistics | Autism Spectrum Disorder (ASD) | NCBDDD | CDC. Retrieved 28 November 2018, from https://www.cdc.gov/ncbddd/autism/data.html

Christensen, D. L., Baio, J., Braun, K. V., Bilder, D., Charles, J., Constantino, J. N.,. . Yeargin-Allsopp, M. (2016). Prevalence and characteristics of autism spectrum disorder among children aged 8 years - Autism and developmental disabilities monitoring network, 11 Sites, United States, 2012. MMWR Surveillance Summaries 2016, 65, 1-23. doi: http://dx.doi.org/10.15585/mmwr.ss6503a1

Constantino, J. N. (2005). Social Responsiveness Scale ${ }^{\mathrm{TM}}$. Los Angeles: Western Psychological Services.

Cooney, B. F. (2002). Exploring perspectives on transition of youth with disabilities: Voices of young adults, parents, and professionals. American Journal of Mental Retardation, 40, 425-435.

Deci, E. L., \& Ryan, R. M. (1985). The general causality orientation scale: Self-determination in personality. Journal of Research in Personality, 19, 109-134.

Fein, D., Barton, M., Eigsti, I. M., Naigles, L., Rosenthal, M., Tyson, K.,...\& Helt, M. (2009). Cognitive and behavioral profiles of children who recover from autism (oral presentation). International Meeting for Autism Research (IMFAR-09). Chicago, IL.

Ghaziuddin, M., \& Zafar, S. (2008). Psychiatric comorbidity of adults with autism spectrum disorders. Clinical Neuropsychology, 5, 9-12. 
Granader, Y. E., Bender, H. A., Zemon, V., Rethi, S., Nass, R., \& MacAllister, W. S. (2010). The clinical utility of the social responsiveness scale and social communication questionnaire in tuberous sclerosis complex. Epilepsy \& Behavior, 18, 262266. doi: 10.1016/j.yebeh.2010.04.010

Grasso, E., Jitendra, A. K., Browder, D. M., \& Harp, T. (2004). Effects of ecological and standardized vocational assessments on office of vocational rehabilitation counselors' perceptions regarding individuals with developmental disabilities. Journal of Developmental and Physical Disabilities, 16, 17-31. https://doi.org/10.1023/B:JODD.0000010037.17733.52

Griffin, C., Hammis, D., \& Geary, T. (2007). The job developer's handbook: Practical tactics for customized employment. Baltimore, MD: Paul H. Brookes Publishing Co.

Griffin, C., Hammis, D., Geary, T., \& Sullivan, M. (2008). Customized employment: Where we are; Where we're headed. Journal of Vocational Rehabilitation, 28, 135-139.

Gulikers, J. T. M., Bastiaens, T. J., \& Kirschner, P. A. (2004). A fivedimensional framework for authentic assessment. Educational Technology Research and Development, 52, 67-86.

Guest, G., \& MacQueen, K. M. (2008). Handbook for team-based qualitative research. Walnut Creek, CA: AltaMira Press.

Hagner, D. (2010). The role of naturalistic assessment in vocational rehabilitation. Journal of Rehabilitation, 1, 28-34.

Hagner, D., \& Cooney, B. F. (2005). I do that for everybody: Supervising employees with autism. Focus on Autism and Other Developmental Disabilities, 20, 91-97.

Happe, F., \& Charlton, R. A. (2012). Aging in autism spectrum disorders: A mini-review. Gerontology, 58, 70-78. doi: 10.1159/000329720

Helt, M., Kelley, E., Kinsbourne, M., Pandey, J., Boorstein, H., Herbert, M., \& Fein, D. (2008). Can children with autism recover? If so, how? Neuropsychology Review, 18, 339-366. doi: 10.1007/s11065-008-9075-9

Hendricks, D. R. (2010). Employment and adults with autism spectrum disorders: Challenges and strategies for success. Journal of Vocational Rehabilitation, 32, 125-134. doi: 10.3233/JVR2010-0502

Hillier, A., Campbell, H., Mastriana, K., Izzo, M. V., Kool-Tucker, A. K., Cherry, L., \& Beversdorf, D. Q. (2007). Two-year evaluation of a vocational support program for adults on the autism spectrum. Career Development for Exceptional Individuals, 30, 35-47.

Holwerda, A., van der Klink, J. L., Groothoff, J. W., \& Brouwer, S. (2007). Predictors for work participation in individuals with an autism spectrum disorder: A systematic review. Journal of Occupational Rehabilitation, 22, 333-352. doi: 10.1007/s10926-011-9347-8

Howlin, P., \& Moss, P. (2012). Adults with autism spectrum disorders. Canadian Journal of Psychiatry, 57, 275-283. doi: 10.1177/070674371205700502

H.R. 803 (113th): Workforce Innovation and Opportunity Act. Govtrack.us. Retrieved from https://www.govtrack.us/ congress/bills/113/hr803.

Hurlbutt, K., \& Chalmers, L. (2004). Employment and adults with Asperger syndrome. Focus on Autism and Other Developmental Disabilities, 19, 215-222. https://doi.org/10.1177/ 10883576040190040301

Jepsen, D. A. (1994). The thematic-extrapolation method: Incorporating career patterns into career counseling. The Career Development Quarterly, 43, 43-53. https://doi.org/10.1002/ j.2161-0045.1994.tb00845.x
Kaufman, A. S., \& Kaufman, N. L. (2004). Kaufman brief intelligence test - second edition (KBIT-2). Circle Pines, MN: AGS Publishing.

Keogh, B. K., Bernheimer, L. P., \& Guthrie, D. (2004). Children with developmental delays twenty years later: Where are they? How are they? American Journal of Mental Retardation, 109, 219-230.

Levy, A., \& Perry, A. (2011). Outcomes in adolescent and adults with autism: A review of the literature. Research in Autism Spectrum Disorders, 5, 1271-1282. doi: 10.1016/j.rasd.2011. 01.023

Lin, J. D., Lin, L. P., \& Wu, J. L. (2009). Administrative prevalence of autism spectrum disorders based on national disability registers in Taiwan. Journal of Autism and Developmental Disorders, 3, 269-274.

Luecking, R. G., Fabian, E. S., \& Tilson, G. P. (2004). Working relationships. Baltimore, MD: Paul H. Brookes Publishing Co.

Maclellan, E. (2004). How convincing is alternative assessment for use in higher education? Assessment \& Evaluation in Higher Education, 29, 311-321. DOI: 10.1080/0260293042000188 267

Mannion, A., Leader, G., \& Healy, O. (2013). An investigation of comorbid psychological disorders, sleep problems, gastrointestinal symptoms and epilepsy in children and adolescents with autism spectrum disorder. Research in Autism Spectrum Disorders, 7, 35-42. doi: 10.1016/j.rasd.2012.05.002

Marriage, S., Wolverton, A., \& Marriage, K. (2009). Autism spectrum disorder grown up: A chart review of adult functioning. Journal of the Canadian Academy of Child and Adolescent Psychiatry, 18, 322-328.

Matson, J. L., Sipes, M., Fodstad, J. C., \& Fitzgerald, M. E. (2011). Issues in the management of challenging behaviours of adults with autism spectrum disorder. CNS Drugs, 25, 597-606. doi: 10.2165/11591700-000000000-00000

McClean, B., \& Grey, I. (2007). Modifying challenging behaviors and planning positive supports. In the handbook of intellectual disabilities and clinical psychology practice (pp. 643-684). New York, NY: Routledge.

Migliore, A., Butterworth, J., \& Zalewska, A. (2014). Trends in vocational rehabilitation services and outcomes of youth with autism: 2006-2010. Rehabilitation Counseling Bulletin, 57, 80-89. https://doi.org/10.1177/0034355213493930

Miles, M. B., \& Huberman, A. M. (1994). Qualitative data analysis: An expanded sourcebook (2nd ed.). Thousand Oaks, CA: Sage Publications, Inc.

Newschaffer, C. J., Croen, L. A., Daniels, J., Giarelli, E., Grether, J. K., Levy, S. E.,...\& Windham, G. C. (2007). The epidemiology of autism spectrum disorders. Annual Review Public Health, 28, 235-258. doi: 10.1146/annurev.publhealth. 28.021406.144007

Nord, D., Stancliffe, R., Nye-Lengerman, K., \& Hewitt, A. (2016). Employment in the community for people with and without autism: A comparative analysis. Research in Autism Spectrum Disorders, 24, 11-16.

O’Brien, G. (2006). Young adults with learning disabilities: A study of psychosocial functioning at transition to adult services. Developmental Medicine \& Child Neurology, 48, 195-199.

Patterson, A., \& Rafferty, A. (2010). Making it to work: Towards employment for the young adult with autism. International Journal of Language \& Communication Disorders, 36, 475480. 
Perkins, E. A., \& Berkman, K. A. (2012). Into the unknown: Aging with autism spectrum disorders. American Journal on Intellectual and Developmental Disabilities, 117, 478-496. doi: 10.1352/1944-7558-117.6.478

Roux, A. M., Shattuck, P. T., Cooper, B. P., Anderson, K. A., Wagner, M., \& Narendorf, S. C. (2013). Postsecondary employment experiences among young adults with an autism spectrum disorder. Journal of the American Academy of Child and Adolescent Psychiatry, 52, 931-939. https://doi.org/10.1016/j.jaac.2013.05.019

Schalock, R. L., \& Keith, K. D. (1993). Quality of life questionnaire. Worthington, OH: IDC Publishing Corporation.

Schalock, R. L., Borthwick-Duffy, S. A., Bradly, V. J., Buntinx, W. H. E, Coulter, D. L., Craig, E. M., \& Yeager, M. H. (2010). Intellectual disability: Definition, classification, and systems of supports (11th ed.). Washington, D.C.: American Association on Intellectual and Developmental Disabilities.

Seltzer, M. M., Shattuck, P., Abbeduto, L., \& Greenberg, J. S. (2004). Trajectory of development in adolescents and adults with autism. Mental Retardation and Developmental Disabilities Research Reviews, 10, 234-247. doi: 10.1002/mrdd.20038

Shattuck, P. T., Wagner, M., Narendorf, S., Sterzing, P., \& Hensley, M. (2011). Post-high school service use among young adults with an autism spectrum disorder. Archive of Pediatrics and Adolescent Medicine, 165, 141-146.

Shattuck, P. T., Roux, A. M., Hudson, L. E., Taylor, J. L., Maenner, M. J., \& Trani, J. F. (2012). Services for adults with an autism spectrum disorder. Canadian Journal of Psychiatry, 57, 284291. doi: 10.1177/070674371205700503

Shogren, K. A. (2013). A social-ecological analysis of the self-determination literature. Intellectual and Developmental Disabilities, 51, 496.

Simonoff, E., Pickles, A., Charman, T., Chandler, S., Loucas, T., \& Baird, G. (2008). Psychiatric disorders in children with autism spectrum disorders: Prevalence, comorbidity, and associated factors in a population-derived sample. Journal of the American Academy of Child and Adolescent Psychiatry, 47, 921-929. doi: 10.1097/CHI.0b013e318179964f

Skokauskas, N., \& Gallagher, L. (2010) Psychosis, affective disorders and anxiety in autistic spectrum disorder: Prevalence and nosological considerations. Psychopathology, 43, 8-16. doi: $10.1159 / 000255958$

Smith, L. E., Maenner, M. J., \& Seltzer, M. M. (2012). Developmental trajectories in adolescents and adults with autism: The case of daily living skills. Journal of the American Academy of Child and Adolescents Psychiatry, 51, 622-631.

Sodak, L. C. (2000). Performance assessments and students with learning problems: Promising practice or reform rhetoric? Reading \& Writing Quarterly, 16, 257-280. https://doi.org/10.1080/105735600406742

Taylor, J. L., \& Seltzer, M. M. (2011). Employment and postsecondary educational activities for young adults with autism spectrum disorders during the transition to adulthood. Journal of Autism and Developmental Disorders, 41, 566-574. https://doi.org/10.1007/s10803-010-1070-3

United States Department of Labor. (2014). Workforce Innovation and Opportunity Act. Retrieved November 22, 2018 from: http://www.doleta.gov/WIOA/

Wehman, P. H., Schall, C. M., McDonough, J., Kregel, K., Brook, V., Molinelli, A.,...\& Thiss, W. (2014). Competitive employment for youth with autism spectrum disorders: Early results from a randomized clinical trial. Journal of Autism and Developmental Disorders, 44, 487-500. doi: 10.1007/s10803013-1892-x

Wilczynski, S. M., Tramell, B., \& Clarke, L. S. (2013). Improving employment outcomes among adolescents and adults on the autism spectrum. Psychology in Schools, 50, 876-887. https://doi.org/10.1002/pits.21718

Williams, J. G., Higgins, J. P., \& Brayne, C. E. (2006). Systematic review of prevalence studies of autism spectrum disorders. Archives of Disease in Childhood, 91, 8-15. doi: 10.1136/adc. 2004.062083

Workforce Innovation Technical Assistance Center. (2017). The Essential Elements of Customized Employment for Universal Application. Retrieved November 29, 2018 from: http://wintac-s3.s3-us-west-2.amazonaws.com/topicareas/Essential-Elements-of-Customized-Employment-forUniversal-Application $\% 20 \% 28005 \% 29$-FInal.pdf

Yin, R. K. (2003). Cast study research: Design and methods (3 $3^{\text {rd }}$ ed.), applied social research methods series (vol.5). Thousand Oaks, CA: Sage Publications.

Ysseldyke, J. E., \& Olsen, K. (1999). Putting alternate assessments into practice: What to measure and possible sources of data. Exceptional Children, 65, 175-185. https://doi.org/10.1177/ 001440299906500204 